\title{
Die Adventsrede des Matthäus de Cracovia vor Papst Urban VI. im Jahre 1385. \\ Von
}

\section{Gustav Sommerfeldt.}

In Pertz's „Archiv" 10, S. 681 hat W. Watten bach bei Beschreibung verschiedener Olmützer Handschriften auch auf 2, VIII, 11 (membr. $4^{\circ}$ ) der Olmützer $k$. k. Studienbibliothek hingewiesen, die $u$. a. eine undatirte Rede des Prager Theologen, spãteren Wormser Bischofs, Matthäus de Cracovia mit dem Incipit ,Quomodo facta est meretrix" entbält. Diese Handschrift, die ich einsehen durfte, stammt aus der bei Olmütz befindlichen ehemaligen Doleiner Karthäuserklause und gehört noch dem 14. Jahrhundert an; die Rede ist fol. 84-93 des Coder enthaiten, und es folgt fol. 94-104 mit der Überschrift "Sermo einsdem magistri Mathei de novo sacerdote, - prelatis nota " eine Synodalrede, die Matthäus zu Prag am 18. Oktober 1384 hielt, und die das Incipit hat, Quid est, quod dilectus meus in domo mea facit scelera multa".

Es gelang mir nun, die erstgenannte Rede noch in drei anderen Handschriften nachzuweisen, zunächst Jagellonische Bibliothek zu Krakau Codex $2244\left(4^{\circ}\right.$, chart. saec. 14) fol. 149-156. Dieser Codex ist in Prag entstanden and wurde 1387 niedergeschrieben, wie eine Eintragung fol. 68 desselben beweist, wo der Schreiber als Datum den 5. Januar 1387 angibt 1). Von derselben Hand, der die Adventsrede in dem Codex verdankt wird, finden sich daselbst noch mehrere Abhandlungen des Matthäus von Krakau, darunter ausser der Synodalrede von 1384 auch zwei andere die Kirchenzucht betreffende Reden, durch die Matthäus auf Geheiss des Erzbischofs Johann von

1) Näheres hierüber: Zeitschrift für Kirchengeschichte 23, S. 595. 
Jenstein den in Prag versanmelten Diözesanklerus zu verschiedenen Zeiten in ernstlicher Weise vermahnte (Zeitschrift für Kirchengeschichte 22, S. 465- 484 und 23, S. 593-615). Eine Hand des 15. Jahrhunderts und eine spätere des 16. Jahrhunderts haben in der Adrentsrede sowohl, als auch in den drei Synodalreden des Codex zahlreiche Rand- und Zwischenbemerkungen angebracht.

Ebenfalls aus dem 14. Jahrhundert stammt die Niederschrift in Bibliotheł der St. Marienkirche zu Danzig Codex 268 (Folio, membr. et chart., ohne Seitenzählung) an der Spitze der zweiten Hälfte dieser Handschrift. - Pelplin, Klerikalseminarbibliothek Codex 40 (Folio, chart), dessen Text fol. 101-105 meist mit demjenigen des Danziger Codex übereinstimmt, gehört dagegen dem 15. Jahrhundert an.

Bei der Herstellung des im Nachstehenden zum Abdruck gelangenden Textes habe ich mich meist des Olmützer und des Danziger Codex bedient'), um beiden Handschriftengruppen so gerecht zu werden. Die abweichenden Lesarten habe ich selten angemerkt, nur am Anfang der Rede in einiger Vollständigkeit wiedergegeben.

Zur Beschreibung des Danziger Codex sei im einzelnen Folgendes bemerkt. Die Niederschrift der zweiten Hälfte des Codex stammt von einem Geistlichen Namens Thomas Glogau her, der den Codex mit den Worten endigt , 0 regina poli, scriptorem linquere noli. Finitus est liber iste per manus Thome Glogaw in vigilia purificacionis sancte Marie ${ }^{4}$. Die erste Hälfte des Codex enthält von anderer Hand des 14. Jahrhunderts den sehr ausführlichen Kommentar eines Ungenannten "super psalmo beati inmaculati in via" mit dem Incipit "Tria sunt, que hominis mentem reddere possunt affectain ad desiderandam noticiam huius psalmi", der Schlnss lautet, ad gaudium de nobis, cum quibus et nos gaudeamus leticia sempiterna, que erit in Christo Jhesu, domino nostro vivente et regnante per eterna secula seculorum, amen". Hinter dem Kommentar sind einige Blätter offen gelassen. Der Schreiber hat hier ein Inhaltsverzeichnis des Codex geben wollen. Es folgen aber nur die am Schluss des Kommentars mit roter Tinte geschriebenen Worte „Registrum super [psalmo] ${ }^{2}$ ) beati inmaculati in via, magistri Mathei etc." Es darf daraus kaum geschlossen werden, dass dieser Kommentar zu Psalm 118 ein Werk des Matthäus von Krakau sei. Denn in Codex Krakau 1312 (Folio, chart. saec. 14) findet sich dieselbe Auslegung dieses Psalms vorerst anonym $\left.{ }^{3}\right)$. Ferner

1) Die Lesarten der Rede in dem Olmützer und dem Rrakauer Codex stimmen bis auf Flüchtigkeitsversehen übrigens fast durchgehends überein.

2) paalmo ist von Thomas Glogau wieder ausradirt.

3) $\mathrm{Vgl}_{\mathrm{g}}$. Wislocki, Catalogus codicum manuscriptorum S. 331. 
enthält Codex C. 178 der Universitätsbibliothek zu Upsala ${ }^{1}$ ) (Folio, chart. saec. 15) fol. 2-88 den gleichen Kommentar mit der Überschrift ,Incipit lectura magistri Mathei de Caracovia super beati inwaculati “, indessen scheint diese Handschrift aus dem Danziger Codex 268 abgeleitet zu sein, und die Čberschrift wohl auf einem Missverständnis jener in den Danziger Codex enthaltenen Registernotiz zu beruben. In der Notiz beziehen sich die Worte ,magistri Mathei" nämlich anf die nach den freigelassenen Blättern in dem Codex unmittelbar sich anschliessende Rede "Quomodo facta est meretrix".

Dieser Rede wiederum sind später von der Hand des Thomas Glogau - ohne Autornennung - zrei Sermone „de nativitate domini" nebst ergäpzenden Notabilien noch beigegeben, und darauf drei Sermone, de assumptione beate Marie virginis gloriose", ebenfalls mit Notabilien ${ }^{2}$ ). Dass dem Matthäus etwas ron diesen Stücken zukomme, ist wegen des sich darin kundgebenden schwerfälligen Stiles und der ungeschickten Argumentation, die sich in den Pfaden der landläufigsten Scholastik bewegt, wenig wahrscheinlich. Zum Teil dürften es Auszïge aus den Schriften des Bernhard von Clairvanx sein, denn am Beginn des dritten der ,Sermones de assumptione Marie" heisst es ron der Hand des Thomas Glogau: „Materiam istam scribit beatus Bernhardus in sermone de assumpcione, qui incipit: filie Jherusalem, nunceiate etc." - Der erste Sermon ,de nativitate domini“ beginnt mit den Worten "Quis te huc adduxit, quid hic agis, quamobrem venire roluisti? Judicum 23. Naturale est, quod novitas effectus", der zweite Sermon beginnt "Queramus domino nostro regi adolescentulam virginem, ut stet coram eo et foreat eum, 3. Regum, 1 ; duo sunt genera hominum ". - Der erste Sermon de assumptione Marie $\left.{ }^{3}\right)$ : Ascendisti in altum, Psal. Quandoque aliquis vult

1) Bei K. Burdach, Vom Mittelalter zur Reformation; Forachungen zur Geschichte der deutschen Bildung. Heft 1. Halle 1893. S. 134 wird die Hundschrift, nach B. Dudík, Forschungen in Schweden für Mährens Geschichte. Bronn 1852. S. 319 noch mit der alten Signatur als Codex theol. 23 zitirt. Eine abweichende, jedoch gleichfalls recht ausführliche, Lectura ouper Psalmum 118 bietet der Krakauer Codex 1428 (Folio, saec. 15, 652 Blatt). Als Schreiber oder Überarbeiter nennt sich darin der Krakauer Theologieprofessor Paul ron Piszkowitz.

2) Den Rest des Codex nehmen verschiedene Schriften des bekannten Richard ron St. Viktor ein.

3) In einer Predigtsammlung, die in Codex Pelplin 142, fol. 161-245 mit der Überschrift ,Postille magistri Mathie de Cracovia de sanctise rorliegt, ist fol. $199 \mathrm{~b}-202 \mathrm{~b}$ eine Predigt, De assumpcione Maries mit dem Incipit ,Omnibos requiem quesivi, Ecclesiastici 24* enthalten. $\mathrm{Zwar}$ ist es nicht sicher festgestellt, ob diese Sammlung von Matthăus von Krakau herstammt, und nicht 
honorare, ponit ipsum in alciorem locum iuxta parabolam ewangelii", der zweite: „Te assumam et regnabis super omnia, que desiderat anima tua, 4. Regum capitalo 11; revolvendo sacre scripture historias", der dritte Sermon: „Maria migratura a corpore decumbebat, ut est infirmitatis humane".

Th. Sommerlad, Matthäus von Krakau, Inauguraldissertation Halle 1891. S. 72 indentifizirt die Rede "Quomodo facta est meretrix" mit einer anderen Rede des Matthäus, die im Krakauer Codex 2244, fol. 74-79 enthalten ist und die Überschrift hat „Sermo magistri Mathei de Cracovia de sanctis apostolis Petro et Paulo, quem fecit in presencia domini Urbani sexti“. Es ist das unzulässig, da der Wortlaut schon äusserlich ein ganz abweichender ist). Aus dem Inhalt der letzteren Rede ergibt sich zudem, dass Matthäus sie an einem Peter-Pauls-Tage (29. Juni) in Rom selbst vor dem Papste gehalten bat, während für die Rede "Quomodo facta est meretrix" ein entsprechender Anhaltspunkt nicht vorliegt, die folgende Erwägung uns vielmehr auf Genua statt auf Rom verweisen wird. In dem Pelpliner Coder 273 (4\% ${ }^{\circ}$. chart., saec. 15) finden sich fol. 205 a-210a "Revelaciones beate Brigitte de Swecia de passione Christi“. Das Incipit lautet: „Inminente tempore Jhesu Christi lacrime erant in oculis eius et sudor in corpore per timorem passionis, bene autem sudor ille saguineus erat", und der Schluss, venit ille bonus Johannes et duxit eam in domum suam; tu auten, domine, miserere nobis". Daran schliesst sich dann von der Hand des Schreibers dieses Stückes $^{2}$ ) der Zusatz „Passio Jhesu Christi collecta per magistrum Mathiam de Cracovia, in sacra theologia magistrum, in civitate Januensi tunc Ronana curia ibidem versante, ex divinis $^{3}$ ) revelacionibus factis beate Brigide

vielmehr von Matthias ron Liegnitz (vgl. Zeitschrift für Kirchengeschichte 23, S. 601); wäre aber Matthăus der Verfasser, so würde das nur einen Beweis mehr dafăr abgeben, dass jene im Danziger Codex enthaltenen Sermone, deren Wortlaut ein abweichender eben ist, nicht von unserem Matthäus herstammen können.

1) Das Incipit lautet: , Quia, sicud ayt Gregorius 6. moralium: qua talibus doctrinne, der Schluss, sanctis apostolis contulit et nobis conferre dignetur dominus Jhesus Christus amen; explicits. - Inlaltlich bietet die Rede wenig von bistorischen Anspielungen. Sie ist aber konkreter gehalten als eine gleichfalls ,de s. Petro et Paulo * betitelte Rede, die des Matthäus Zeitgenossen, den Pariser Kanzler Johannes Gerson zum Verfasser hat. Vgl. über diese J. B. Schwab, Johannes Gerson; eine Monographie. Würzburg 1858. S. 391.

2) Die Uberschrift von anderer Hand und in etwas blasserer Tinte. Ein sich fol. 210 unmittelbar anschliessender Traktat, de ieiunio e wird anonym gegeben.

s) Wiolocki, Catalogus, in Beschreibung der Handschrift: ,collecta ex diversis revelacionibus", doch ist diversis 2 weifellos verlesen. Birgittas Visionen 
per dominum Jhesum Christum et per Mariam virginem gloriosam et partim per angelum in sermone angelico etc."

Handschriftlich ist dieselbe "Passio" auch enthalten in Codex 171 des Krakauer Kapitelsarchivs 1 ) und in Codex 1399, fol. 184 a-187 b der Jagellonischen Bibliothek zu Krakau, an letzterer Stelle mit der Überschrift ,Collectum de libris Brigitte, que babuit de passione domini per revelacionem etc." In diesem Codex kommt zwar der Name des Matthäus von Krakau bei der Passio nicht unmittelbar vor, es schliesst sich aber die Passio an des Matthäus ebenda fol. 145̃a-184a vorausgehenden Traktat "de passione domini“ an, der das Incipit hat „Feria quarta post festum palmarum“, und endigt, singulariter diligit, Johannis 15, qui diligit etc." Dieselbe Passio nun liegt ferner in einem Antwerpeuer Druck vom Jahre 1489 vor, wie G. E. Klemming, Heliga Birgittas uppenbarelser (in: Samlingar utg. af Srenska fornskrift-sällskapet). Bd. V. Stockholın 1883. S. 203 näher dargetan hat ${ }^{2}$.

Es hat im Pelpliner Codex nicht etwa Verwechselung mit Matthias, Domherrn von Linköping, dem Beichtrater Birgittas, stattgetunden, sondern es handelt sich um den Prager Professor Matthäus von Krakau, dessen Beziehungen zum Birgittenkult auch sonst genügend bekannt sind (Sommerlad S. 16). Ond ferner wird bei Klemming V, S. 202, was erst recht entscheidend ins Gewicht fällt, Matthäus von Krakau als Verfasser eines auf Birgitta bezüglichen Sermons genannt, der mit anderen verwandten Stücken zu Rom im Jahre 1485 durch Magister Encharius Franck im Druck herausgegeben wurde ${ }^{3}$ ).

Da Papst Urban VI. nur in der Zeit vom 23. September 1385 bis 16. Dezember $138 b^{\circ}$ seine Hofhaltung in Genua hatte, ergibt sich genannter Stelle des Pelpliner Codex zufolge, dass ein Aufenthalt des Matthäus von Krakau bei der päpstlichen Kurie in eben jene Jahre

schieden sich in solche, die ihre Anbänger als divinae oder divinitus factae bezeichneten und zweitens in visiones diabolicae. Vgl. H. Lundström in Realencyklopädie für protestantische Kirche und Theologie III (Leipzig 1897), S. 239244 und J. Fijalek, Mistrz Jakób z Paradyža Bd. II. Krakau 1900. S. 102.

1) Ig n. Polkowski, Katalog rękopisów kapitulnych katedry krakowskiéj. Teil I. Krakau 1884. S. 114.

2) Der Drucker ist Gerhard Leeu. - Eine Neuauflage ohne Angabe des Druckorts erschien 1491, und eine Neubearbeitung: Köln 1517 (Kle m ming $V_{\text {. }}$ S. 205); ferner Holländische Übersetzungen der Passio gedruckt zu Antwerpen 1491 und Amsterdam 1506 (K le m m ing V, S. 227-229).

3) Franciks bezügliche Publikation führte den Titel, Onus mundi, aliquerevelationes quarte libri celestium revelationum sancte Birgitte (K) lemming a. a. O. V, S. 200-2021. 
zu setzen ist. Und da Matthäus die Rede "Quomodo fucta est meretrix" nach Ausweis des Wortlautes derselben als Adventsrede gehaliten hat, so folgt weiter, dass dieselbe in den Dezember 1385 zu setzeu ist. Denn 1386 kann nicht in Frage kommen, da Matthäus nachweislich am 18. Oktober 1386 aus Anlass der Prager Proviuzialsynode ssich noch in Böhmen befand, und ferner eine in Dezember 1386 gehaltrene Rede nicht mehr in dem Krakauer Codex 2244 hätte Aufnahme fiuden können, von dem oben nachgewiesen ist, dass er Anfang Januar 1387 niedergeschrieben wurde.

Nicht der geringste Grund aber liegt vor, sie mit Sommerllad

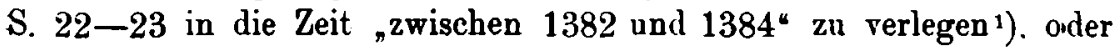
mit $\mathrm{Finke}^{2}$ ) in die Zeit ,um $1382^{*}$.

Dagegen kann die oben erwähnte Rede de Petro et Paulo, die Matthäus vor dem Papste in Rom an einem Peter-Paulstage hielt, zu zirka 1382 angesetzt werden. - Der Zweck der Entsendung des Matthäus im Jahre 1385 wird nicht derjenige gewesen sein, den Papst der Ergebenheit der Prager Universität zu versichern - solches hatte Matthäus schon früher getan, als er vor demselben Papst die Rede, de sanctis Petro et Paulo" hielt -, sondern er wird einen "Rotulus" der Oniversität Prag überbracht ${ }^{3}$ ) und zugleich dem Papste über die im Jahre 1384 an der Prager Universität unter dem Rektorat des Konrad vou Soltau vorgefallenen antideutscheu Unruhen berichtet haben ${ }^{4}$ ).

Einen Mitgesandteu des Matthäus bei seiner Mission vom Jahre 1385 haben wir wahrscheinlich in den Heinrich von Hannover zu sehen, der als „magister in artibus studii prefamosi Pragensis" zu Genua im Jahre 1386 auftritt in Handschrift 5 der Bibliothek des Marienstiftsgymnasiums zu Stettin (fol. $369 \mathrm{a}-394 \mathrm{a})^{5}$ ). Leider ist die

1) Vgl. J. Losertb, Hus und Wiclif; zur Genesis der Husitischen Lehre. Prag 1884 S. 68, Anw. 5, wo einige Auszüge aus unserer Rede gegeben werden.

2) H. Finke in Wetzer und Welte's Kirchenlexikon VIII, S. 1039 (Frei. burg 1893).

3, Uber die Bedcutung dieses Gebrauchs siche L. Schuitz, Kourad von Soltau. Jena. Dissertation. 1891. S. 18.

4) Schmitz a. a. O. S. $11-13$.

5) Th. Lind n er. Geschichte des deutschen Reiches vom Ende des 14. Jabr. hunderts bis zur Reforwation. Bd. I. Braunschweig 1875. S. 253, Anm. 1 sagt, dass König Wenzel [im Jahre 1386] mehrfach Gesandte nach Italien geschickt hake, nenut aber keine Quelle. aus der dic Namen dieser Gesandten ersichtlich wären (vgl, auch G. Erler, Theoderici de Nyem de scismate libri 3. Lipsiae 1890. S. 128). - Heinrich von Hannover beendigte zu Genua am 3. Dezember 1386 ein im Stettiner Codex 5 (siehe fol. 394a) enthaltenes grammatisches Werk, Quaestiones et sophismata circa Donatum (H. L e m cke, Die Handschriften und alten Drucke der Bibliothek des Marienstiftsgynnasiums zu Stettin. Teil I. Stettin. 
Persönlichkeit dieses Heinrich von Hannover nicht sicher festzustellen. Denn wenn Lemcke) ihn mit Heinrich von Einbeck (auch Embeck und von Nanexen genannt), Domherrn zu Mainz, identifiziren möchte, der als Professor der Prager Universität wiederholt genannt wird ${ }^{2}$ ). so ist hiergegen einzuwenden, dass Einbeck im "Liber decanorum facultatis philosophicae universitatis Pragensis“ von Eude März 1372 ${ }^{3}$ ab nicht mehr genannt wird. An Heinrich von Oyta, den berühmten Rechtsgelehrten, kann erst recht nicht gedacht werden. Dieser siedelte, nachdem er 1378 Prag verlassen und seinen Aufenthalt in Paris genommen batte, 1383 nach Wien über4).

Nicht ganz unwahrscheinlich will es nir unter diesen Umständen vorkommen, dass mit Heinrich ron Hannover etwa der Prager Magister Henricus de Bremis gemeint sein könnte, der in den 80er Jahren in Prag oft genannt wird, und 1392 zum Rektor der Prager Universität gewählt wurde ${ }^{5}$ ).

Gymn.Programm. 1879. S. 7). Neues Material über die Beziehungen der Kurie zu Deutschland in diesen Jahren brachten bei H. V. Sa uerland im, Historischen Jahrbuch < 14, S. 820-832 und J. K a u f m a $\mathrm{n} n$ in, Quelleu und Forschungen aus Italienischen Archiven und Bibliotheken Bd. II, S. 285-306 und III, S. 69-81, indessen wird auch bej ihnen der Name des Heinrich von Hannover nicht genannt. Vgl. noch L. Zanutto, Il cardinale Pileo di Prata e la sua prima legazione in Germania, 1378-1382. Udine 1901.

1) Lem ke a. a. O. S. 7, Anw. 21.

9) W. Tomek, Geschichte der Prager Universität. Prag 1849. S. 14 und 354. In Codex Krakau 2244 ist fol. 98-104 unter Einbecks Namen eine undatirte ,Collatio * (Gelegenheitsrede bei Kircheinweihung) überliefert mit dem Incipit ,Pax huic domuie. Die Meinung bei J. A. I abricius, Bibliotheen Latiua mediae et infimae aetatis. Bd. III. Hamburg 1735. S. 638, dass Heinrich ron Einbeck auch den Namen, Engelus e geführt und 1430 zn Herdegessen bei Göttingen gestorben sei, scheint willkürlich zu sein und allein auf unsichern Notizen Tritheims zu beruhen

8) Monumenta historica universitatis Carolo-Ferdinandeae Pragensis. Bd. I. Prag 1830. S. 151.

4) J. Aschbach, Geschichte der Prager Universität. Bd. I. Wien 1865. S. 403, Fij.alek a. a. O. I, S. 38: u. If, S. 65 , H. De n ifle, Die Universitäten des Mittelalters. Bd. I. Berlin 1895. S. 592, H. V. Sa u erland, Rede der Gesandtschaft des Herzogs Albrecht III. von Österreich an Papst Lirban Vl., circa 1387 (Mitteilungen des Instituts für österreichische Geschichtsforschung 9, S. 448). - Der Heimatort ist Oyta in Ostfriesland.

s) Monumenta hist. univ. Prag. I, S. 104. 
Sermo magistri Mathei de Cracovia coram papa t cardinalibus in curia predicatusci).

- Quomodo facta est 8 ) meretrix civitas fidelis? Ysaie 1. In venerabili ac.3). venerabiliter formidanda culminis apostolici et sancte Romane curie predicare presencia) res est difficilis et pregrandis ${ }^{5}$ ). Nam cum omnis acra scriptura divinitus inspirata atilis sit ad docendum, arguendum, erudiendom et increpandum ${ }^{6}$ ), Thimot. 2, quis est, qui vel cogitare andeat se velle docere tocius mundi magistrum, arguere vel docere ${ }^{7}$ ) universorum ${ }^{8}$ ) correctores simal et iudices, vel presumere, quod sint ${ }^{9}$ ) in aliquo corrigendi. Rursum si $^{10}$ ) in ecclesia dei aliquid mali esso vel periculi inminere perpenditur, ubi, queso, periculosins ${ }^{11}$ ) dici poterit, quam coram eis, quoram interest 12) fidelinm obviare periculis et providere saluti 13). Ant quis iustios annuncciabit ${ }^{14}$ ) quam is, qui summa et apostolica anctoritate datus esse videtur in speculatorem domni Israhel. Unde et timere habet, qui in hoc loco predicat, ut ${ }^{15}$ ) vel se ostentare vel placere cupiens, mala ecclesie et pericula fidelium non annunceiati6), et miram est, si non timet, ne de manibus suis requiratur sagwis non solum audiencium, qui de tanto excusari videntar ${ }^{17}$ ), quanto arduis occopati ${ }^{18}$ ), nec singulis ${ }^{19}$ ) potentes intendere, volunt habere et procurant babere ${ }^{20}$ ) viros litteratos, qui eos hortentur, prenunccient ${ }^{21}$ ) et instigent ${ }^{22}$ ) -, sed eciam sagwis eorum, de quorum salute procuranda 23 ) ipsos sollicitare debuerat ${ }^{24}$ ). Quod si quis animam suam liberare volens annuncciare voluit, o quanta opus habet discrecione et providencia 25 ) coram mundi luminaribus, ne de errore arguatur, quanta reverencia, humilitate et decencia verborum, ne ostentacionem querere et presumptuosus ac temerarius videatur, quanto pondere et maturitate verboram, ne velle placere, et per hoc Christi servas non esse probetur. Quanta talium auditorum benignitate et clemencia, ut, quod dictum fuerit, benigne ${ }^{26}$ ) recipiant ${ }^{27}$ ) et ad benigniorem partem interpretentur, quia ${ }^{28}$ ) inpossibile est aliquando ${ }^{29}$ ) in multa et ardua dicendo esse tam providum, qui possit effugere instancias et obiecciones ${ }^{30}$, si

1) Im Krakauer Codex (K) lautet die Utberschrift: ,Sermo magistri Matbei de Cracovia curiensis coram papa im Olmützer Codex $(0)$ : ,Sermo, quem fecit magister Matheus 8. de Cracovia, sacre theologie doctor, coram Urbano papa sexto, cum esset ambasiator studii Pragensis" [W atten bach a. a. 0 . unrichtig: , cum ceteris ambasintoribus $\left.{ }^{\star}\right]$. $O b$ das in 0 hinter Matbeus folgende s. als eine Verkürzung für , stadtschreiber', den Familiennamen des Matthäus (vgl. S o mmerlad 8. a. O. S. 13-15), anzusehen wäre, möchte ich nicht für gewiss ausgeben.

2) D (Danzig) und $\mathbf{P}$ (Pelplin): es; $\mathbf{K}$ Strich über e.

3) $\mathrm{K}$ : formidabili etc. corain venerabili et formidabili culmine sedis apostolice et sancte Romane curie predicare etc.

b) KU: corripiendum.

$\begin{array}{ll}\text { 8) } \mathrm{O} \text { : universis. } & 9 \text { ) sint fehlt in } \mathrm{P} \text {. } \\ & \end{array}$

ii) KO hinter periculosins: tacebitur vel fructuosius. ${ }^{12} \mathrm{~J}$ : est.

19) $\mathrm{K}$ überschrieben de salutiferis. 14) $\mathrm{KO}$ : annunctiabit. . 15) 0 : et.

16) EO: annunctiat. 17$) \mathrm{DP}$ : videtur, 0 : excusati videntur.

11) DP: occupari. 19 ) K: simul. 20 ) babere fehit KO.

2) $\mathrm{K}$ : premuniant.

24) DP: debuerant.

27) $P$ : accipiant.

29) 0 : aliquem.

*? $) \cdot \mathrm{K}$ : instigant.

95) DP: prudencja.

2s) $K$ : quasi eniw, $O$ : quasi non inpossibile.

30) $\mathrm{K}$ : obiectus. 
remota pietate faerit iudicatus. Ego igitur videns tot hinc inde pericula, necessarium habeo orare dominum, ut det sermonem rectum et bene sonantem in os meum, Hester 14. Rectum ') quidem, ut non nisi dicenda loquar, bene sonantem, ut dicta benigaius acceptentur. Ut autem hoc multipliciter intercessoribus ${ }^{2}$ ) facilius inpetretur, omnes, queso, mecum orare dignemini et matrem gracie salutare salutacione angelica, dicentes ave Maria. - Quomodo facta est meretrix etc. Adventum domini ${ }^{3}$ ) omnes adesse novimus secundum tempus et ecclesie institucionem; sed secundum maliciam temporis, que hactenus fuit ${ }^{4}$, et ecclesie, si dicere licet, destitacionem magis videtur esse tempus recessus dominici quam adventus. Vel si dici debet, adventus, conveniencius dicetur antichristi quam Christi. Vel si omnino contenditur ${ }^{5}$ ), ut Christi dicatur adventus, pocius ${ }^{6}$ ) dicetur ad vindictam venire, quam ad graciam. Etenim si discessio ${ }^{7}$ ) signom est adventus antichristi, vel dei ad iudicium ${ }^{8}$ ), que evidencior discessio quam que nostris diebus facta est, in qua tam multi antichristi facti sunt, ut, si quis infidelium converti velit ${ }^{9}$ ) ad (hristum, non de facili sciat, quo diverti, aliis dicentibus: ecce hic est Christus, aliis ${ }^{10}$ ): ecce illic, at dominus Matb. 12 futurum esse predixit11). Licet antem dens nunquam derelinquit sperantes in se, sed cum ipsis sit eciam in tribulacione, et quanto minus ecclesiam sponsam suam electam vel dilectam ${ }^{12}$ ), tamen quia tam multi eam deserunt, et nos tam in multis derelinquit ${ }^{13}$ ) in die tribulacionis et in tempore superborum sine adintorio, qnid alind convinci potest, nisi quod dominus ipse, qui sibi appropinquantibus appropinquat, Jacobi 4: appropinquate deo, et appropinquabit vobis, et ut ait beatus Augustinus, neminem deserit, nisi deserstur, desertus a nobis eciam nos deserat et derelinquat. Unde verbam assumptum quandam nostri separacionem a deo et spirituale divorcium innuens, et si non esset ad propositum tempore currenti, secundum nomen convenit tamen malicie temporis nunc presenti. Verumptamen et tempori et nomini 14) congruit ${ }^{15}$ ), quia omnium ${ }^{16}$ ) contrariorum eadem est disciplina. Dum de adrentu agitur, de recessu non incongrue disputatur. Pro aliquali igitar assumpti thematis applicacione ad propcsitum et eius iutrodnccione considerandum, quod superamabilis et excellentissima divine bonitatis inmensitas, quamvis illam caritatem perpetuam, qua nos dilexit et attraxit miserans, et flagrantissime ${ }^{17}$ ) sue mirifice caritatis ardorem maltipharie multisque modis exhibere dignata sit, pariter et monstrare18), nunquam tamen tam. evidenter apparuit ille superfervidus ac intimus dulcissimi amoris affectus, sicud quando sponsus esse et sibi sponsam assumere dignatus est, dignabitur et ${ }^{19}$ ) dignatur, quod in tribus demonstratur adventibus ${ }^{20}$ ), videlicet

i) 0 : Tercium.

Rand wiederholt de adventu.

fuerit.

b) $\mathrm{K}$ : extenditur

2) P : multiplicibus intercessionibus.

s) in $\mathrm{K}$ am 4) $\mathrm{R}$ versatur überschrieben, $0:$ actenus 6) KO: melius.
discessio quam fehlen in $\mathrm{DP}$.

${ }^{8}$ ) in $\mathrm{K}$ überschrieben : extremı iudicii. o) KO: wnlt.

10) $P$ : alius.

ii) $K$ am Rande: eo, quod quidam dicunt, hec est fides, alii autem asserunt contraria. 12) vel dilectam fehlt in $O$.

14) P: tempore et nomine.

15) 0 : convenit:

ii) KP : flagrantissimam.

18) $\mathrm{K}$ : convenit. dileccio maxime ostenditur in triplici adventu Christi.

$\left.{ }^{20}\right) \mathrm{K}$ : communiter descriptum atque adventibus, $\mathrm{O}$ : convincitur adventibus.
25 
in carnem, in animam et ad iadicium. Sane in primo adventa ${ }^{1}$, quando pater fecit nupcias filio, Math. $23^{2}$ ), quibus in cubilis) virginalis uteri mirifice celebratur, in assnmpta natura egressus est tanquam sponsus procedens de thalamo suo, Psalmo 18. Quantam ibi caritatem monstraverit, ex eo constat, quod, ut ait beatus Augustinus in libro de predestinacione sanctorum, non erat, quo alcius eveberetur humana natura, quam quod deas homo fieret. Unde Wilhelmus de fide et legibus: sicud gatta ${ }^{4}$ ) aque ad mare magnum et uns ${ }^{5}$ ) scintilla ad ignem occupantem totum mundum, sic omnia dei beneficia prins exhibits ad hoc ${ }^{6}$ ) lumen, nec in boc contentas illam caritatem, qua, ut dicit Johannes 14, maiorem caritatem nemo habet, exhibuit ponens animam suam pro amicis ${ }^{7}$ ) suis. Inde et de latere ${ }^{8}$ ) morientis in cruce per aquam mundam et saguine ${ }^{9}$ ) decorata sponsa formaretur ecclesia, sicut de latere Ade dormientis formata est Eva mater nostra. In secundo adventu ${ }^{10}$ ) per graciam in animam, quo conpletur illa desponsacio Ozee 2 promissa: sponsabo te mihi in eternum, sponsabo te mihi in iudicio et insticia, in miseria et miseracionibus, et sponsabo te mihi in fide. Magnitudo caritatis inde colligitur ${ }^{1}{ }^{1}$ ), quia tunc homo adoptatar in filium, stola prima induitur, annulus in manu ${ }^{12}$ ) et calciamenta in pedibus dantar ${ }^{13}$ ). Accipit anima racione ntens primicias spiritus, gustat et videt, quam suavis est dominus, fruitar ipso et fruendo delectatur, unitur sibi et in ipsum transformatur, et sic adherens deo unus spiritus fit cam eo, 1. ad Corinth. 6. Unde beatus Bernardus super cantica sermone $7:$ non sunt inventa eque dulcia nomina, quibus verbi animeque dulce 3 adinvicem ${ }^{14}$ ) exprimerenta ${ }^{\circ}$ affectus, quemadmodum sponsus et sponsa, quippe quibus omnia communia sunt, nil proprium, nil a se divisum habentibus. Una utriusque hereditas, una $\operatorname{dos}^{15}$ ), una mensa, unas thorus ${ }^{16}$ ), una caro. - Porro in tercio ${ }^{17}$ ) adventu ad iudiciam, quando in ${ }^{18}$ ) medis nocte clamor fiet: ecce sponsus venit, exite obriam ei, et que parate erant, intraverunt cum eo ad nupcias, Math. 25. - Illas inquam ${ }^{19}$ ) nupcia3, de quibus Apocal. 19: venerant nupcie sponse et agni, quibus assumit sibi ${ }^{20}$ ) sponsam ecclesiam gloriosam non habentem m sculam neque ragam, nec aliquid haiasmodi, Ephes. $5^{21}$ ). - In hoc, inquam, adventu seu nupciis, nedum exhibicio sed tocius caritatis consummacio plena fiot a deo, ut beati dicantur, qui ad cenam nupciarum agni vocati sunt, Apocal. 19. Eece quantum nos secum unum adinvicem esse voluit, quos in se, et quious tam salubriter per assumpłam naturam, tam dulciter per infusam graciam, tam feliciter per promissam gloriam uniri proposuit, ut satis appareat, quantum 22) delicie sue sint esse cum filiis hominum. Sicut autem tanti sponsi sponsam esse vel fieri ${ }^{83}$ ) magnam dei dignacionem et summam

1) K am Rande: de primo adventu: tunc humana natura est des desponsata in perpetuum, et salus nostra est inchoata.

für die Zahl. 3 ) $\mathrm{KO}$ : cubiculo. $\quad$ i) $\mathrm{P}$ : gutte.
6) $\mathrm{K}$ : adbuc.
7) $\mathrm{KO}$ : ovibus.
8) $\mathbf{K}$ : ut inde latere.

o) PO: sagwinem.

10) $\mathrm{K}$ am Kande: de secundo: tunc homo adoptatur in filium dei et heredem, nostra salus innovata.

12) 0 : manus.

15) $K$ : unus deus. 16, $\mathrm{K}$ : unum corpus; am Rande zugefügt: vnus
thorus.

13) $\mathrm{K}$ : induantur. i1) P: tollitu:.

11) 0 : et ad idem.

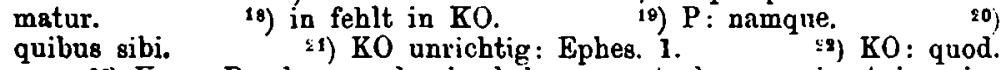

so) DP: in

23) $\bar{K}$ am Rande: quod, sicud bonum est, desponsacio et iunccio hominis cum deo, sic pessima est eius separacio et adulterio ab to. 
creatare dignitatem ac exaltacionem explicat, sic econtra in maximsm redundat iniuriam, iramque indignacionis sue vehementissime provocat, turpissimam quoque reddit creaturam, ut ad extremum vilescat, si tam amabili sponso contempto alteri nupserit et ${ }^{1}$ ) frangendo fidem se prostituerit meretricem, ita quol tam turpis mutacio digna omnino videatur magna ammiracione, dura exprobracione, dira lamentacione. Quis enim non ammiretar ${ }^{2}$ ) potenciam discretivam illustratam superno lumine sic infatuari, at dicat bonum malum, malum bonum, ponens tenebras lacem et lncem tenebras, Ysaie 1. Quis non detestatur potenciam nobilissime preditam $^{3}$ ) divino munere tantum depravari, ut derelinquat deum creatorem suum. Quis non ${ }^{4}$ ) lamentetur potenciam ${ }^{5}$ ) memorativam recreatam ${ }^{6}$ ) interna dulcedine sic amaricari, ut experimento cognoscat, quam malum et amarum est dereliquisse dominam deum snam, Jerem. 2. - Sed audiamns venersbilem Anshelmam?) in deplanctu virginitatis amisse, animam susm alloquentem ${ }^{8}$ ) in hec verba: tu, inquit, o anima mea, que quondam désponsata deo in baptismo virgo ${ }^{9}$ ) fuisti, ta, inquit, perfida deo, periura deo, alultera Christi, tu illi prior libellum repudii obtulisti, ta te sponte ad dyabolum convertisti? 0 quam pium, quam amabilem dereliquisti, quam impio et quam borribili te coniunxisti! Dereliquisti in celo castum amatorem tuum et amplexata es horridum corraptorem et crudilissimam tortorem tuum. 0 quam de sublimi cecidisti, quam in profundum te diraisti, o quid fecisti, o quantum perdidisti. 0 miserrimum perdere illum, illud bonum, denm perdere irrecupersbiliter, quem servare debueras interminsbiliter; hec ille. Cam igitur hoc interrogatum, quomodo hec tria significari reperiatur in scripturis, ammiracionem, at Johannis 12: mirabantar Judei dicentes, quomodo hic litteras scit, cum non didicerit; exprobracionem, ut Ysaie 14: quomodo cecidisti lucifer, qui mane oriebaris ; lamentacionem, ut in Trenis 1: quomodo sedet sola civitas. Hinc est, quod propheta ammirando racionis sic decepte demenciam, detestando voluntatis sic allecte maliciam, deplorando memorie sic infecte miseriam, de qualibet anima peccatrice ${ }^{10}$ ), que et civitas dici potest, Jeremie $1:$ dedi te in civitatem munitam. Querit exprobrative ${ }^{1}$ ), quomodo facta est meretrix civitas fidelis. - Si autem nnius anime malicia tam mirabilis, tam exprobrabilis, tam lamentabilis esse decernitur, quanto magis lapsus innumerose multitudinis vel pote ${ }^{12}$ ) parate et integre civitatis, quis et ipsa sponsa est, Apocal. $21^{13}$ ): vidi civitatem sanctam Jherusalem descendentem a deo, paratam sicut sponsam, ornatam viro sno, presertim ubi tot sunt circumstancie aggravantes, videlicet tam firmi et securi status amissio, quia civitas, Proverbiis 18: frater fratrem adiuvans quasi civitas firma, tam radicate et quasi murate 14) fidei violacio, quia fidelis, 1. Corinth. 2: dampnacionem babentes, quia primam fidem irritam fecerunt, tam vilis statas acceptacio, quia meretrix, Jeremie 2: quam vilis facts es nimis, iterans vias tuas, et tante pravitatis consummacio, quia facta est meretrix,

\footnotetext{
1) 0 : id est.

2) P: ammiracione. tatain. 4) creatorem - non fehlt in KO.

3) KO: volitivan predoB) $\mathrm{K}$ : vim.

6) 0 : recreata.

7) $\mathrm{KO}:$ sed in venera

8) $\mathrm{KO}:$ ulloquitur.

9) $\mathrm{KO}$ : et virgo.

Anshelmo in planctu.

i) $\mathrm{K}$ : obprobriosa querela, $\mathrm{O}$ : oprobriosum querelis.

19) DP: unrichtig : 5.

14) DP: murate unitatis.

10) P: meretrice.

iq) KO: ut pute.
} 
non quod primo incipiat vel in conplendo sit, sed in esse conpleto, Trenis 4 : conpleta est malicia tua, filia Syon, propter quod dicit ipse deus, cui nomen est zelotes, Exod. 34. Zelo zelans pro sponsa filii sui pater, inquit, noster, qui $\Theta$ st in celo, merito exprobrando querit et obicit: quomodo facta est meretrix etc. - In quibus quidem verbis duo considerari possumt: mocio viciosa et questio dubiosa. Tangitur enim mocio viciosa, qua dei sponsa $\operatorname{tam}^{1}$ ) graviter ceciderit, quis facta est meretrix etc. Queritur quoque ${ }^{2}$ ) facti forma, et qualiter acciderit, cum dicitur: quomodo. - Casus nimis $^{3}$ ) stultus atque reprobandas, et ob hoc ignominiose proponitar, modus multis occultus et investigandus, et ideo studiose inquiritar. Casu? proponitur ignominiose, ut pre confusione moneatur ad resipiscendum. Modus inquiritur studiose, ut ex monicione inducatur ad se cognoscendum. Dico primo, quod tangitur mocio viciosa, qua dei sponsa tam graviter cecidit ${ }^{4}$, pro quo notandum, quod humane salutis amator et auctor volens dignitatem hominis existentis in gracia describere et nos ad puritatis virtutem ${ }^{5}$ ) ac castum sui amorem allicere, omnem creaturam racionalem, hic in via, ant $^{6}$ ) animam fide per dileccionem operantem preditam nobilissime puritatis nomine virginem ac delicatissimi amoris vocabulo sponsam voluit appellari, dicente apostolo Corinth. 11: despondi ${ }^{i}$ ) vos uni viro virginem castam exhibente Christo, et ex adversa parte volens vilitatem peccatoris ${ }^{\star}$ ) exprimere et nos ad detestacionem peccati et fugam inducere, omnem creaturam racionalem, $u^{9}{ }^{9}$ animam illa fide et dileccionis puritate carentem nomine horribili et despectissimo meretricem appellare decrevit. Unde Jeremie 2 dicitur: in omni colle sublimi, videlicet per superbiam et ambicionem bonoris, et sub omni ligno frondoso per delectacionem, scilicet inordinatam, tu prosternebaris ${ }^{10}$ ) meretrix. Ft que gravior matacio, quam de tante dignitatis honore, de tante decore mundicie, de statu sponse, de tam desiderabilium amenitate deliciarum, ad tante feditatis horrorem, vilitatis opprobrium, al statum tam abhominabilem et despectum cadere et mutari11). Non est hec ${ }^{12}$ ) mutacio dextre excelsi, sed casus luciferi dampnabiliter execrandus, $\Lambda$ pocal. 17: veni, ostendam tibi dampnacionem meretricis magne, et $\left.18^{18}\right)$ : cecidit Babilon illa magna, et facta est habitacio 14) demoniorum. Sicut autem sponsam illa tria substancialia ${ }^{15}$ ) faciunt: matrimonii fidei promissio, boni prolis intencio et inseparabilis unio, sic revera meretricem reddit cuiuscunque eorum, maxime si pro pecunia fiat, transgressio. Quamvis autem tante nomen turpitudinis omni homini conveniat, si plus quam deum temporalia querat, diligat vel intendat, iuxta illud beati Gregorii $\left.{ }^{16}\right)$ : cave, o anima nea, ne non sponsa sed meretrix dicaris, si munera dantem ${ }^{17}$ ) plus quam amantis affec-

1) KO: Tangitur enim dei sponsa, quam graviter.

9) $0:$ quomodo.

s) $\mathrm{K}$ : minus.

6) $K \mathrm{am}$ Rande: Sicnd anima existens in gracia propter dileccionem dicitur virgo et sponsa Christi, sic propter peccatum dicitur meretrix et adultera.

7) $\mathrm{P}$ : desponsavi.

s) $0:$ puritatem virtutum.

8) $\mathrm{KO}:$ peccatorum.

6) DP: ut.

10) $K$ : prosternaberis.

11) 0: imitari.

9) KO: aut.

18) DP unrichtig : 1.

i4) D unrichtig abhominacio.

12) $\mathrm{K}$ : hic.

15) $\mathrm{K}$ ain

Rande: que faciunt hominem sponsam et meretricem spiritualiter respectu Christi.

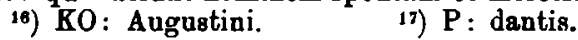


tum diligis, clericos tamen principaliter temporalia querentes ${ }^{1}$ ) gravius concernit. Qui sicut se spiritualiter et pre aliis deo desponsant et dedicant, sic amplius bonum prolis intendere, et ut multos filios per ewangelium in Christo gignant, continue debent parturiendo laborare. Sed, queso, quis status in ecclesia, que dignitas, quis grailus scienciarum ad sacrorum²) ordinum assumitur officium ${ }^{3}$ ), quis actus exercetur, in quo non, ut convincitur, magis lucrum ${ }^{4}$ ) pecuniarum et sustentacionem ${ }^{5}$ ) corporum, quam ad dei gloriam et salutem animarum intendatur. Nonne hoc conqueritur dominus Malach. 1: quis est, inquit, in vobis, qui claudat bostia et incendat altare meum gratuito? Quid aliud sentit propheta dicens Psalmo 78: posuerunt, inquit, Jherusalem in pomorum custodiam, id est in desertum ${ }^{6}$ ) collocatis pomis. An non idem testatur apostolus Ephes. $2^{7}$ ), dicens: omnia, que sus sunt, querant, non que Jhesu Christi. Unde beatus, ut quidam volunt, Bernardus, sed secundum alios Gilbertus Poretanus in sermone 3 ${ }^{8}$ ), can. 3: quesivi et non inveni illum. Querunt, inquit, multi, et si non ipsum Jhesum, siquidem de nomine Jhesu tractatur in consiliis ${ }^{9}$ ), disceptatur in iudiciis, cantatur in ecclesiis, disputatur in scolis. Sed vade ad exitus viarnm et vide, quis horum generaliter est finis operum, vide, si non in omnibus hiis quedam exerceantur mercimonia. De Christo questuosa est res, nomine Christi nil preciosius, quia nil optacius, et de beneficiis, ut verbis beati Bernardi utar ${ }^{10}$ ), quis ea intencione querit, nec, ut debet, ymmo queritur? Queri enim et non querere deberet. Unde cuilibet talium Ezechiel $16^{11}$ ) dicitur: ad omne caput vie edificasti signum prostitucionis tue. Quid enim vie, nisi illud ${ }^{12}$ ), quod est inicium accionum nostrarum. Primum in intencione signum ergo prostitucionis ad omne capnt edificat, qui hoc verbis vel factis dat intelligere, quod in omnibus, que agit, plus querat lucrum temporale, quam ad alind quippiam intendat. Quod si apostolns illos adulterantes iudicat, qui verbum dei non sincera intencione predicant ${ }^{13}$ ), dicens 2. ad Corinth. 2 : non sumus sicut plurimi adulterantes verbum dei, sed ex sinceritate loquimur ${ }^{14}$ ). Cur non et illos adulterare dicemus, qui beneficia recipiunt et fructum facere non intendunt, qui non solum peceare se sciunt, sed sponsam dei decipinnt. Sed eciam timeant se ad omnium reddituum, quos isto modo percipiunt, restitucionem obligari15). Sicut enim, qui in desponsacione carnali in bonum prolis rel ad alind, quod est de substancia matrimonii, ex proposito non consentiret, non sponsus sed adulter vel fornicarius per copulam carnalem fieret, bonis femine ateretur iniuste et ad restitucionem eorum, que in hoc proposito perdurans perciperet, teneretur. Sic et multo magis in matrimonio spirituali videtur, si essenciales condiciones eiusdem excluduntur, que sunt deam diligere, Johannis ultimo. $\mathrm{Si}$ diligis me plus hiis, et fructum facere,

1) $\mathrm{K}$ am Rande : quod clerici spiritualiter dicuntur transgressores, meretrices

$\begin{array}{lll}\text { et adulteri. } & \text { 2) } \mathrm{P} \text { : sacerdotum. } & \text { 5) } \mathrm{KO}: \text { quod officium. }\end{array}$ 7 ) in $\mathrm{D}$ offen gelassen. в) $\mathrm{K}$ : desercionem. $P$ : in desertis collectis pomis. statt 1, 0: superi, 1 can. 3. beati Bernardi utar. $\quad$ i1) DP unrichtig: Exodus $16 . \quad 19) \mathrm{K}$ statt Quid 9) P: conciliis. beati Bernardi utar. 11 i) DP unrichtig: Exodus 16.119$) \mathrm{K}$ statt Quid

10) D: hec ille : illo - illud : Si enim vie, ut ait Gregorius, sunt acciones nostre, quid est capuó vie nisi id. is) P: obligare.

18) $\mathrm{K}$ am Rande: de beneficiatis.

14) 0 : loquamur. 
Johannis $1^{1}$ ): ego vos elegi, ut eatis et fructum afferatis etc., presertim quia bii, qui non intendunt Christam, non intrant per hostium, et per consequens fures sunt et latrones, Johannis 10: fures quidem per occultim maliciam intrando, latrones bona ecclesie aperte tollendo. Sed quis dubitet fures et latrones ad restitacionem teneri ${ }^{2}$ ), cum peccatom non dimittatur, nisi restitustur ablatam ${ }^{3}$ ). Sed quid miri, si alie acciones nostre prostitunntur, cum et ipsa fides, que signaculum est spiritaslis virginitatis, proch dolor sit venalis. 0 quot et quante ${ }^{4}$ ) fuere civitates fideles, que illam prostitnerunt, quot ecclesie kathedrales, quot collegia, quot monasteria religiosorum, quot civitates et castella domino nostro pro vero papa et vicario Jhesa Christi tenendo fảem debitam denegant et adherent antipape ob hoc maxime, ne temporalia bona perdant. Si hic cam propheta deberem fodere parietem, abhominabilia viderentur, quod principes et prelati seculares et viri ecclesiastici, expresso eciam, ut dicitar, pacto precedente, precio conducti sunt, ut antipape adhererent ${ }^{5}$ ) ad maximam adversariorum confusionem, qui fidem hominum pecunia estimant possideri. Numquid non in eis conpletum est illad propheticum Ezechiel 166): to autem dedisti mercedes cunctis amatoribus tuis et donabas eis, ut intrarent ad te ad fornicandum tecum; factumque est in te contra consuetudinem mulierum: in eo, quod dedisti mercedes, non accepisti, et o utinam illi solum ${ }^{7}$ ), et non aliqui eciam de nostris, venderent fidem suam. Vereor, omnino vereor, quod non omnis, qui dicit, domine, domine ${ }^{6}$ fidelis est domino nostro ${ }^{8}$ ), quodque nonnulli sunt, qui secum tenent verbo, sed animo tenent partem oppositam, et ${ }^{9}$ ) aperte foverent, si non sperarent, huic ${ }^{10}$ ) vivendo vel inherendo. lucrari, vel timerent, illuc ${ }^{11}$ ) recedendo privari. Hii, si meretrices dici non debent, dicantur eis peiores, utpote a regno dei remociores. Sic enim ait dominus quibnsdam simulantibus veritatem et non amantibus, Math. 21 : meretrices et publicani precedent vos in regno dei. Nec mirum, quia simulats equitas est duplex iniquitas. Unde tales, qui vel spe lucri vel damni timore domino nostro vel parti adherent adverse, pulchre fgurati videntar per illas mulieres, que at due meretrices ${ }^{12}$ ), ut dicitur 3 . Regum 3, venerunt ad regem Salomonem. Ille enim in domo una babitabant, et isti in diversis ${ }^{13}$ ) divisa unitate ecclesie permanent ${ }^{14}$ ). Illarum ${ }^{15}$ ) una flium suum opprimens, alteri vivam furtim abstulit et apposito illi mortuo sibi vivum fallaciter usurparit. Ista una pars, scilicet nobis adversa, veritatem et iusticiam derelinquens, et per hoc Christum ${ }^{26}$ ), qui datas est nobis filius, in cordibus suis per peccatum opprimens ${ }^{17}$ ), nobis errorem inponit, et sic filium in nobis esse mortuum et in eis vivere mendaciter asserit et contendit. Sed eam novercam et non veram matrem ${ }^{18}$ ) demonstrat illa vox maligna, novercitatis signnm, testis invidie: nec mihi nec tibi, sed dividatur, dum hinc invidia, illinc avaricis stimulante magis.
1) $P: 16$.
2) DP: non teneri.
3) $\mathrm{K}$ am Rande: de Hussitis.

4) DP: quanti.

5) $K$ am Rande: $a b$ obediencia ecclesie Romane

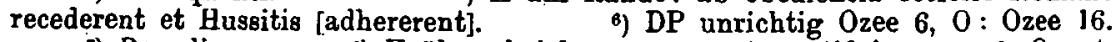

7) P! soli.

10) $\mathrm{K}$ : hic, 0 : hinc.

8) $\mathrm{K}$ aberschrieben: verum et pontifici.

9) 0 : ut.

mulieres aut duas meretrices, $O$ fehlt ut due meretrices.

14) $O:$ permanebant, $K$ : in diversa unitate ecclesie propinabant.

15) DP: Illa. ${ }^{16)} \mathrm{P}$ : exemplum.

17) $\mathrm{KO}$ : interimen?.

18) 0 : matrem esse. 
elegerint, qued tanica Christi inconsutilis, ymmo Christus ipse divisus esset, per hoc quod nedum de baptismo, sed eciam omnium sacramentorum et sacrorum percepcione zelus et contencio est, alio dicente: ego sum Urbani, alio ego Clementis, vel verius dicendo dementis, quam quod elegissent unicaem in vincalo caritatis et pacis in omnibas ${ }^{1}$ ) salvativam, quia deus caritas est, et qui manet in caritate, in deo manet, et deus in eo, 1. Johannis 4. 0 abi fuit tunc et ubi adbuc hodie est illa vox pia, vox magis eos deeens ${ }^{2}$ ) et toti mundo congruens, vox materne fidei, pietatis vox materne, vox viscerose conmocionis et dileccionis uterine ${ }^{3}$ ): date illi infantem vizum, et non dividatur vel moriatur. Vox, inquam, consideracione digna, qua alteri pia mater ${ }^{4}$ ) magis elegit honore materno et cordis sui comere solacio, de biis favere alteri et quasi negare naturam, quam divisionem filii videre vel mortem. Discant prelati, qui sponse Christi et fdelium matres esse volunt et debent, nec pudeat eos eciam a tali matre discere, quanta agere debeant pro vitanda morte filiorum et salute obtinenda ${ }^{5}$ ). 0 quis mihi det videre diem istum, ut omnes meretrices convertantar in matres, hoc est, at omnes, qui querant honorem temporalem ant consolacionem ant lucrum, principaliter et maxime vitam et salutem desiderent animarnm. Credo enim, propter quod et loquor, quia statim esset finis scismatis ${ }^{6}$ ), si extinctom esset desiderinm temporalis comodi ${ }^{7}$ ) vel honoris ${ }^{8}$ ). 0 dolor, o plus quam dolor et miseria, 0 pador et ignominia, inorror et insania, quod propter miserabile bonam temporale tam diu durare debet ant sinitur tam dampnabile molum et periculum spirituale. Et hoc de primo.

Dixi secundo, quod in verbis premissis notari potest questio dubiosa, qua queritar facti forms, et qualiter acciderit, cam dicitar: quomodo 9 ). Quia enim tantum ${ }^{10}$ ) malum est in ecclesia dej, cuius vix aliqua pars tacts est, et cui modis omnibus remedium esset adhibendum, merito studiose inquiritur et studiosissime inquirenda est causa morbi, qui nunquam bene curabitar, nisi radicitus ipsa radix precidatur, quia, ut ait Gregorius in 2. pastoralinm: Incassum foris ${ }^{11}$ ) nequicia ex ramis inciditur, si surrectura multiplicius in radice servetur. Constat antem clernm presencinm ${ }^{12}$ ) malorum precipuam esse causam, tam ex evidencia facti, quam scriptura. Quis enim fecit scisma 13) nisi sacerdotes, quis sic persecntus est dominum nostrum, at clerici, per quod tamen principum ${ }^{14}$ ) excusacionem non pretereando ${ }^{15}$ ), quorum nonnulli sunt infideles, socii furam, diligentes munera etc., que dicuntur Ysaie 1. Constat eciam ex testimonio scripturarum in eis, at pote morbi radice ${ }^{16}$ ), adhibendam esse medelsm exemplo salvatoris, qui, postquam deplorasset maliciam Jherusalem et miseriam predixisset, intravit in templam et corripait sacerdotes, ut patet Lace 19 et Math. 21. Unde Crisostomus super Matheum hoc idem tractans sic ait: hoc erat boni medici, ut ingressus ad

$\begin{array}{lll}\text { 1) } \mathrm{KO} \text { : filii in hominibus. } & \text { 2) } 0 \text { : docens. } & \text { s) unitive. }\end{array}$

4) 0 : quam alteri mater. $\quad$ s) 0 : tenenda, $K$ : radicaliter cognito.

-) $\mathrm{K}$ überschrieben: male, dei perfidie. nostris. 8) $K$ uberschrieben : in Bohemis.

9) $K$ am Rande: quod multa mala sunt in ecclesia, quorum clerus est causa precipua. 10) fehlt in 0.

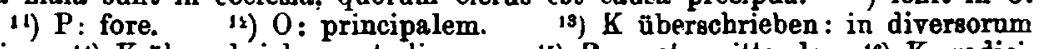
heresim. 14) $\mathrm{K}$ überschrieben: et aliorum. 13) $\mathrm{P}$ : pretermittendo. 16) $\mathrm{K}$ : radici. 
infirmam civitatem sanandam ${ }^{1}$ ) primo ad originem passionis sue mente ${ }^{2}$ ) tenderet. Nam sicut de templo omne bonum, sic et omne malum procedit, quemadmodum medicus quando nrimum ingreditur ad infirmum, statim de stomacho eius interrogat et eum conponere festinat. Qui si sanus fuerit, totum corpus validum est, si autem disparatus ${ }^{3}$ ), tutum corpus ${ }^{4}$ ) infirmum. Ita si sacerdocium integrum fuerit, tota ecclesis floret, si autem corruptum fuerit, omnium ${ }^{5}$ ) fides est marcida; et paulo post: vere, inquit, quemadmodum si videris arborem pallentem foliis, marcidam intelligis, quia aliquam culpam habet circa radicem, ita cum videris populum indisciplinatum et irreligiosum, sine dubio cognosce, quod sacerdocium non est ${ }^{6}$ ) sanum. Hec ille. Huic concordat illud Ysaie 4: venerunt structores tui destruentes te et dissipantes te. Sed cum quasi innumerabiles sint defectus et mala clericorum, ob hoc incurabilis esse videatur morbus, nisi radix eius spiritualius ostendatur. Quo, queso, alio modo deficere videatur ecclesia, nisi recedendo a viis ${ }^{7}$ ) patrum, qui eam plantaverunt, et modum deserendo ${ }^{8}$ ), quo crevit. Quis enim dabitet facilius eam conservari per illa media inter tot amicos et fideles ei assistentes, per que crevit inter tot tyrannos et infideles undique persequentes. Quia vero fuerunt in primitiva ecclesia multa protunc utilia, que pro nunc vel non sunt, vel non congruunt, ut miracula, gracia9) ligwarum, paupertas et similia, sufficerent ad reformandum ecclesie statum hec tria essencia ${ }^{10}$ ), si rectores ei lucerent doctrina salntari, eminerent vita exemplari, ferverent zelo regulari. Hec enim tria data fuerunt apostolis et signis exterioribus designata: ligwe loquentis ${ }^{11}$ ), ignis lucentis pariter et ardentis. Unde hec tria defecisse in ecclesia deplangere videtur Jeremias in Trenis 4, velut ${ }^{12}$ ) si questioni nostre respondere volens dicat: queris, quomodo facta est meretrix, et quasi respondens dicit: eo scilicet modo, quo obscuratum est aurum, mutatus est color optimus, dispersi sunt lapides sanctuarii in capite omnium platearum. Obscuratum est enim aurum sapiencie radiantis, qua deberet doceri veritas et intellectus illustrari ad recti congregacionem. Mutatus est color optimus vite edificantis, qua deberet moveri voluntas et affectus inclinari ad boni imitacionem. Dispersi sunt lapides sanctuarii a fervore zeli regulantis, quo deberet coherceri pravitas et defectus emendari ad mali repressionem. Primum ergo, per quod deficit ecclesia, est, quod obscuratum est aurum sapiencie ${ }^{13}$ ), presertim in rectoribus et presidentibus, ex quo provenit defectus doctrine salutaris. Dum enim semen subtrahitur, procul dubio omnis fructus impeditur ${ }^{14}$ ). Semen autem est verbum dei, Luce 8. Semen, inquam, eciam ipsius fidei ${ }^{15}$ ), que fundamenlum est tocius edificii spiritualis, dicente Johanne in Apocal.: fides ex auditu, auditus autem per verbum Christi. Quod

2) KO: salvandam.

2) fehlt in $K$.

4) fehlt in 0 , $K$ extat statt corpue.

6) UP: habet.

o) 0 : genera.

7) DP: ab biis.

10) $\mathrm{K}$ : tamen ad reformandam ecclesiam hec tria ewangelica, und an hande: tria sufficerent ad refornacionem ecclesie, que aperte in eadem deficiunt, quia prelati non lucent doctrina salutari.

12) $\mathrm{K}$ uberschrieben: de quo. prelati non lucent doctrina salutari.

16) $\mathrm{K}$ überschrieben: predicacio.
(I) 0 : lucentis. 14) DP: ipso dicente statt impeditur. 
autem ex defectu sajiencie deficit ecclesia'), testatar beatus Bernardus pulchre dictum approbans in libro de amore dei: 0 felices, inquit, res bumanas ${ }^{2}$, dicit quidam philosophus, si aut ${ }^{3}$ ) soli sapientes regnant aut philosophentur, qui regnant. Sed cum sapienter fugiunt sapientes regnare, et insipienter fugiunt insipientes erudiri4), omnia dissipantur ${ }^{5}$ ), omnia confunduntur et conturbantur. Sapientes latent et delitescunt ${ }^{6}$ ), pueri ${ }^{7}$ ) regnant et principantur, et fiunt principes, qui mane conmedunt, sed ve terre illi! Hec ille. In magno ergo et faciliter provideretur ecclesie, si in conferendis dignitatibus et beneficiis, in officiis et potestate conmittendis ${ }^{\varnothing}$ ) sapientes ${ }^{9}$ ), dum aliud canonicum non obsisteret vel obstaret, omnibus aliis preferrentur, sicut et de iure deberent preferri. Tnnc enim quilibit sive nobilis sive plebeius anhelaret ad studium, et feret multitudo sapientum ${ }^{10}$ ) sanitas orbis terrarum, Sapiencie 6. Subtraheretur eciam occasio multis malis, ne scilicet iuvenes tempus ${ }^{11}$ ) iuventutis studio aptum in lascivia et vanitate consumerent, qui iam videntes plures promoveri per servicia et promociones principum, quam pericia ${ }^{12}$ ) litterarum, oceasionem habent aut sumunt libencius adherendi curiis quam stadiis, vel magis proficiendi in baratra quam in theologia, et sic dies suos consumendi, quod eis iustius beneficia negarentur quam donentur. Etenim non habens scienciam sit nobilis, sit in obsequio fidelissimas, sit in temporalibus summe providas, habeat omnium promociones principum, inhalibis tamen est et secundum àeum a sacerdocio vel saltem animaram cura est repellendus, dicente domino per Ozeam prophetam 4: quia repulisti scienciam, repellam et ego te, ne sacerdocio fungaris mihi. Maxime cum ille sollempnis doctor Henricus de Gandano ${ }^{13}$ ) 13. canone, questione 14 dicat, illos caratos esse in statu dampnacionis, qui predicare nesciunt, aut si sciunt predicare, non tamen predicant secundum indigenciam populi tempore oportuno ${ }^{14}$ ). Cai quidem doctori ${ }^{15}$ ), et si plus assenciendum quam dissenciendum estimo, magis tamen eorum, quorum interest, determinacioni relinquo, quam assero. Sed est, quod quis sciat docere ${ }^{16}$ ) et preesse valeat, adhuc, sicut idem solempnis doctor nonnullis interpositis subdit, minus ydoneus ad curam animarum, quando ${ }^{17}$ ) videt, quod magis ydonens haberi potest, fugere debet, si potest, ut magis ydoneus ${ }^{18}$ ) preferatur; eciam certe racionale videtur, alioquin deo gloriam et popalo profectnm subtrahit, quem magis 5 donens efficere poterit super ipsam. Quantam igitar diligenciam collatores beneficiorum et provisores debent habere, ne alios in statu dampnacionis ponant, et pereant simal cum eis et ipsi. Sed quia, dum male vivitur, deturpatur sciencia et doctrina contempnitur,

1) KO : ex defectu sapientum mundus pereat. sapientibus servierint insipientes, sed et felices res humanas.

4) $\mathrm{KO}:$ sub sapientibus esse.

cescunt propter impedimentum.

8) DP: conmittentes.

10) $\mathrm{K}$ üherschrieben: quibus consiatit.

12) $K$ : sine pericia, $P$ : per periciam.

i4) KO : pro tempore oportuno.

17) KO : animarum existens, cum.

fehlen in $\mathrm{K}$; am Rande jedoch: debet cedere.
7) $\mathrm{K}$ aberschrieben : pueri vero.

2) 0: si ubique

9) DOP : autem.

6) $\mathrm{K}$ : delus. 
ideo secunduw, quo ${ }^{1}$ ) deficit $^{2}$ ) ecclesia, quod ${ }^{3}$ ) mutatus est color optimus vite exemplarist). In quo licet magis difficulter posset provideri quam in primo, turpe tamen est et inexcusabile, si tali conmittatur cura animarum et $^{5}$ ) ecclesia, cui non conmitteretur pauca ${ }^{6}$ ) pecania, aut si minor diligencia habeatur de bono pastore animarum, quam de fideli procuratore expensarum. Quis enim sponsam dilectam capitali suo et sponse inimico, aut oves suas lupo conmitteret? Omnis autem malus sacerdos est inimicus dei et lupas in oves testante beato Dyonysio in epistula ad Demophilum: profecto cecidit a sacerdotali ordine, qui non est illuminatus sciencia, et magis, qui non est illustratus gracia. Non est enim sacerdos, sed inimicas dolosas, delusor sui ipsins et lapus super dominicum populum pelle ovina armatus ${ }^{7}$ ). Sed quia eciam sapientes, saltem quo ad scienciam agendam ${ }^{8}$ ), negligant et propter impedimenta malornm boni a bonitate deficinnt, et $o b$ hoc similiter correccione indigent, ideo tercium malum est ${ }^{9}$ ), per quod defectus in ecclesia fiunt, quia dispersi sunt lapides sanctuarii et tepent a fervore zeli regularis. Quis enim non videat, quanta essent in ecclesia dei exterminanda, corrigenda et reformanda 10). Exterminande forent hereses et secte, corrigendi mali et quasi omnes status reformandi ${ }^{11}$ ), ordines scilicet et monasteria, universitates et studia, ecclesie kathedrales et collegia, civitates et oppida, principes et prelati, rectores et subditi, iudices et adrocati, sacerdotes et laici, qui omnes declinaverunt, et tam inutiles facti sunt, nt non sit, qui faciat debitum et servet statum suum. Et nos dispersi propter temporalia hiis omnibus obmissis totum tempus impendimus circa lites et iurgia, redditus et beneficia, boni provisores, qui de summe necessariis nullam et de superfluis vel minimis maximam gerimus curam. De illorum quidem sumus disposicione, conservacione et multiplicacione solliciti, quorum sollicitudinem dominus probibnit, Math. 6: nolite solliciti esse dicente etc. ${ }^{12}$ ), sed omnium talium sollicitudinem in ipsum proiciendam esse voluit. Quantum ipsi est cura de nobis, et illorum sollicitudinem postponimus, quam requirit a nobis et precipue ad Ephes. $4^{13}$ ): solliciti servare unitatem spiritus in vinculo pacis, et Michee 6: indicabo tibi, o homo, quid sit bonum, et quid deus requiret a te, facere indicium et iusticiam et sollicitum ambulare cum domino deo tuo ${ }^{14}$ ). O quanta boni servi facimus, que probibet; et que iubet, non curamus. Dum autem sic servimus, ut volumus, ipse eciam pro velle suo retribuit, dimittit siquidem nos ire ${ }^{15}$ ) in adinvencionibus nostris. Et quis contra doctrinam apostoli prudentes fuimus ${ }^{16}$ ) apud nosmetipsos, ipsam stultam faciet sapienciam nostram, ut illa via perdamus bona temporalia, qua ea conservare et acquirere nitebamur. Accidetque nobis conformitanter, ut Judeis, qui timentes, ne locum et gentem perderent, principem Christum occiderunt et per eandem occasionem omnia

1) 0 : ideo in quo.

2) DP: in ecclesia.

s) $\mathrm{K}$ : est quia.

4) K am Rande: constare poterit ex predictis, de secundo periculi, quia prelati non eminent vita exemplari, que vita valde viluit in clericis et licet in $\begin{array}{ll}\text { isto. } & \mathrm{KO} \text { : vel. }\end{array}$ hec ille. $\quad$ s) $K$ am Rande: interdum.

7) $\mathbf{K}$ überschrieben : periculi, guode $\mathrm{K}$ an Rande: de tercio periculi, quod prelati non fervent zelo regulari, quo multa feri deberent in ecclesia.

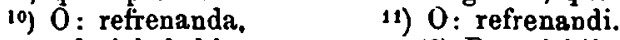
qui conmederi habebimus. 13) $P$ unrichtig: 3.

18) $\mathbf{K}$ überschrieben : $\begin{array}{lll}\text { amicum. } & \text { 15) } \mathrm{KO} \text { : ire et perire. } & \text { 16) } \mathrm{K} 0 \text { : sumus. }\end{array}$ 14) $0:$ sollicitum 
perdiderunt. Sic et nos, dum pacem cum malis, quam Christus non venit mittere in terram, querentes, $\mathrm{ac}^{1}$ ) principum et dominorum ${ }^{2}$ ), ne temporalia nobis anferrent vel auferant, favorem conservare volentes, iusticiam obmittimus, multa mala et iniusta dissimulamus, et per hoc Christum, qui est veritas, Johannis 14, et qui factus est nobis insticia, 1 Corinth. 1, in cordibus multorum per negligenciam nostram mori facimus, quod accidit certe in eis Christo mortuo. Nec sentencias excommunicacionis timent nec clerum advertunt, sed audacter invadunt, thelonea et alias exacciones ab eis recipiendo, libertates infringunt ac in miserabilem ${ }^{3}$ ) redigunt servitatem, bona eorum tenendo et de illis pro suo beneplacito disponendo ${ }^{4}$ ). Et qui sunt dyocesani, qui seniencias excommanicacionum aut interdicti ferie vel sentencias exequi audeant contra tales! Si antem Christam et eius glorism intenderemus, et verbo, exemplo aut zelo ferventi filios spirituales iteram et iterum parturiremus, donec formaretur in eis Christus, tanc ipsi Christo babito et dilecto $^{5}$ ), Christianos saos targere et in prophetis malignari timerent ipso Cbristo in eis hoc prohibente. Quod enim preter Christum habemus, non bene ${ }^{6}$ ) per ipsum poterimus conservare, quoniam, qui contempnit deum, vicarium non curabit. Hec omnia per prophetam Psalmo 88 confirmantur brevi verbo. Et non audivit, inquit, populus meus vocem meam, et Israhel non intendit mihi. Eece servicium, en meritum nostrum, et sequitur statim premium. Et dimisi eos secandam desideria cordis eornm ${ }^{7}$ ). Quod antem, si sibi intenderemus, et ipse nos defenderet, mos ostendit, cum dicit: si populus me andisset, Israbel si in viis meis ambulasset, pro nichilo forte inimicos eorum humiliassem, et super bona eorum misissem manum meam. - Qua propter, reverendissimi et metuendissimi domini mei, det nobis saltem ipsa vexacio demum nostri ${ }^{8}$ ) intellectum, ut intendamus dilecto nostro, ut et ipse nobis intendat iuxta illud Cantici 6 : ego dilecto meo et dilectas mens mihi, et assumamus zelum pro nomine sancto eius ac sponsa dilecta, ne habeat conqueri de nobis illud 1. Regum $20^{9}$ ): non est, qui doleat vicem meam ex robis. Quis enim nostrum est, qui videns sponsam fratris sui debere stuprari, non prohiberet, si posset, vel saltem contradiceret et doleret. Et ecce Christus, qui non confanditar fratres nos vocare, et plus esse quam frater, ipse dominus, ipse pater, ipse crestor ${ }^{10}$ ) et consultor, ipse cibus refeccionis et redempcionis precinm, retribucionis preminm, ipse totum bonum nostrum, qui, ne frustrs et solo nomine celebremus adventum suum, nobis advenire dignetur per graciam et tandem nos sumere secum ad gloriam, quam nobis concedat ' 1 ) deus pater et dominas filins et spiritus sanctus. Deus unus in secula benedictus, amen.

Die persönlichen und literarischen Beziehungen des Matthäus von Krakau dürften trotz der bisher gemachten Versuche noch nicht in hinreichender Weise aufgeklärt sein. Meine Erwartung war, hierüber

1) P: actis.

4) KO: dando.

2) K tiberschrieben: secularium. s) $\mathrm{K}$ : dileccione.

7) KO: ibunt in adinvencionibns suis. solator. s) $0:$ miserias.

6) K: nisi, 0: enim.

8) demum nostri fehlt $\mathrm{KO}$.

10) KO: creator, conservator, redemptor et coni1) 0 : concedat etc. - Die Worte deus - amen fehlen in 0 . 
Näheres aus einem „Epistolarum liber I ad diversos" zu ersehen, den Sommerlad S. 75 unter den Schriften des Matthäus von Krakau nennt. Der betreffende Codex der Breslauer Universitätsbibliothek I F 272 (Folio, chart. saec. 15) hat aber überhaupt vichts ron Matthäus von Krakau '), sondern enthält fol. 1-39 des Kardinal Bonaventura "Liber interne consolationis" und fol. 40-105 „Mathei Vegii Laudensis" 7 Bücher, de perseverantia religionis ad Elizabeth et Monican sorores von Ende des 15. Jahrhunderts. Dagegen bieten, was weder bei Loserth noch bei Sommerlad angemerkt ist, Codex Breslau I F 273, fol. 100-101 und Codex Breslau I F 286 fol. 139140 eine undatirte Epistel dar, in der Matthäus von Krakau ,quamvis valde invitus" aus Anlass eines bestimmten ihm zur Beantwortung unterbreiteten Falls die Stellung erörtert, die der Christ im geschäftlichen wie im allgemeinen Leben dem Judentum gegenüber einzunehmen hat. Die Überschrift in Cod. I F 273 lautet: „Incipit epistola scripta per magistrum Matheum de Cracovia sacre theologie doctorem, episcopum Wornaciensem, et directa archiepiscopo Pragensi contra carnifices, quod non debeant participare Judeis". - Adressat des Briefes ist Zbynèk von Hasenburg, der 1403-1411 das Errbistum Prag hatte, wenn es richtig ist, dass Matthäus zur Zeit, als er len Brief verfasste, schon Bischof von Worns war. Da indessen Codex I F 273, der ursprünglich aus Neisse stammt, im Jahre 1406 geschriejen ist, wie eine Notiz in demselben fol. $186 \mathrm{~b}$ ergibt, und da femer Matthäus im Jahre 1405 erst Bischof geworden ist, dürfte der Brief einer älteren Zeit zuzuweisen sein und auf Matthäus' Gönner, len schon genannten Prager Erzbischof Johann von Jenstein gehen.

1) Veranlasst sind die Ausführungen Sommerlads durch eine irrige Notiz bei J. Loserth, Hus und Wiclif S. 69, Anm. 1, der in jenem Breslauer Codex , Epistolae" des Matthäus von Krakau enthalten glaubte. 